\title{
Reversible myelopathy: an uncommon accompaniment of hypothyroidism
}

\author{
Nimisha Jain ${ }^{\mathrm{a}, \mathrm{b}}$, Ramesh Aggarwala,b, Sachin Kumar Jain ${ }^{\mathrm{a}, \mathrm{b}}$, Nitin Garg ${ }^{\mathrm{b}, \mathrm{c}}$, \\ Aadhaar Dhooriaa,b, Kajal Prasad ${ }^{\mathrm{b}, \mathrm{c}}$
}

aDepartment of Medicine, Lady Hardinge Medical College and Associated Smt S.K.

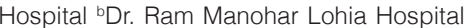
'PGIMER, New Delhi, India

Correspondence to Ramesh Aggarwal, MD, Department of Medicine, Lady Hardinge Medical College and Associated Smt S.K Hospital, New Delhi, 110001, India

e-mail: rameshlhmc@gmail.com

Received 9 February 2014

Accepted 24 April 2014

The Egyptian Society of Internal Medicine 2014, 26:86-87

\begin{abstract}
Atypical neurological presentations of hypothyroidism include myelopathy which has been seldom reported as presenting feature in patients. This case was one of those atypical presentations of hypothyroidism in which patient presented with neurological complication viz myelopathy which was timely diagnosed and treated. The symptoms including neurological findings reversed with thyroxin replacement. The case highlights the varied and vivid manifestations of hypothyroidism which treating physicians should be aware of while treating patients.
\end{abstract}

\section{Keywords:}

atypical presentation of thyroid, hypothyroidism, myelopathy

Egypt J Intern Med 26:86-87

(C) 2014 The Egyptian Society of Internal Medicine

$1110-7782$

\section{Introduction}

Hypothyroidism is usually diagnosed clinically on the basis of its typical symptoms of weight gain, hoarseness of voice, and delayed reflexes on examination. Neurological manifestations such as muscular weakness, nerve entrapment syndromes, peripheral neuropathy, cold intolerance, ataxia, dementia, and convulsions may accompany the typical presentations or sometimes precede other symptoms of hypothyroidism. Myelopathy has rarely been described in the literature as a presenting complaint for patients of hypothyroidism. This patient presented with hoarseness of voice along with weight gain, but surprisingly he also had exaggerated reflexes and extensor plantars. These features suggestive of myelopathy soon reversed when patient was treated with thyroxin.

\section{Case report}

A 40-year-old man, who was driver, nonsmoker, and occasional alcoholic, presented with weakness of both lower limbs since last 5 days. He was unable to stand from squatting position and his footwears slipped from his feet. He did not complain of any sensory loss but could not stand or walk straight. Later, he confessed that he had been gaining weight and his voice was becoming unclear for the last 3 years. General physical examination was unremarkable except bradycardia, mild nonpitting edema over shin, and coarse dry skin. His speech was hoarse; power in both lower limbs was $4 / 5$; and reflexes were exaggerated. Babinski sign was present bilaterally. Gait including tandem walking was impaired, and the Romberg test was positive. No other significant finding could be elicited in other systemic examination. Most of the clinical examination was suggestive of hypothyroidism except upgoing plantar in both lower limbs. Blood investigations were suggestive of macrocytosis with hemoglobin of $13.5 \mathrm{~g} / \mathrm{dl}$ and MCV of $112 \mathrm{fl}$. Thyroid profile proved this case as suffering from hypothyroidism with thyroid-stimulating hormone of $444 \mathrm{mIU} / 1(0.5-5)$, FT3 of $0.5 \mathrm{pg} / \mathrm{ml}(2-4.4)$, FT4 of $0.07 \mathrm{ng} / \mathrm{dl}(0.6-2.2)$, and anti-TPO antibody of $12 \mathrm{IU} / \mathrm{ml}$ (20-50). Vitamin $\mathrm{B}_{12}$ and folate levels in blood were almost normal, that is, $174 \mathrm{pg} / \mathrm{ml}$ and $2.9 \mathrm{ng} / \mathrm{ml}$, respectively. Other investigations including chest radiography and ECG were normal. MRI of the spine could not be performed because the patient was not able to afford the cost.

\section{Discussion}

The prevalence of hypothyroidism is estimated to be around $3.9 \%$ in India, with an estimated 42 million people suffering from thyroid diseases [1]. Hypothyroidism is usually diagnosed by its common clinical manifestations such as weight gain, constipation, fatigue, menorrhagia, dysarthria, and cold intolerance. Rarely, certain neurological features such as decreased psychomotor activity, dysarthria and hoarseness, muscle and joint pain, cramps or stiffness, muscular weakness, nerve entrapment syndromes, peripheral neuropathy, cold intolerance, ataxia, dementia, and convulsions may be the presenting complaints when it becomes difficult for the clinicians to suspect hypothyroidism [2]. This patient, despite having features of hypothyroidism, 
had atypical neurological signs such as exaggerated reflexes in the lower limbs and presence of Babinski sign. The close differentials such as vitamin $B_{12}$ deficiency, transverse myelitis, or compressive myelopathy were ruled out by clinical and biochemical examination. Bilateral extensor plantar with exaggerated reflexes without cortical involvement and without definitive sensory level still suggested myelopathy as the underlying mechanism with hypothyroidism as probable etiology. The patient was empirically initiated with thyroxin $(100 \mu \mathrm{g})$, and in the next 7 days he improved remarkably and his plantars became flexors.

There are few case reports in the past where patients with hypothyroidism developed encephalopathy and myelopathy. These patients had Hashimoto's thyroiditis, and the presence of thyroid antibody was associated with development of neurological problems. This patient diagnosed as suffering from severe hypothyroidism probably developed myelopathy, which was reversible soon after replacement with thyroxin. However, unlike previously reported cases antithyroid antibodies were not present in this patient, and the mechanism responsible for reversible myelopathy remained unclear. Association of thyroid antibodies with encephalopathy was first proposed by Brain et al. [3] in a patient with Hashimoto's thyroiditis. Although the exact mechanism still remains unclear, they postulated the development of cerebral edema causing encephalopathy in their patient. Subsequently, in another report, the development of myelopathy preceded encephalopathy in a patient with Hashimoto's thyroiditis [4].

\section{Conclusion}

This case highlights two key features:

(1) Hypothyroidism can sometimes present with atypical neurological features such as myelopathy as present in this patient.

(2) Empirical treatment with thyroxin can treat hypothyroid myelopathy and improve neurological features in such patients.

(3) Myelopathy without the presence of antithyroid antibodies has not been mentioned in the literature, and therefore this case will help in further research for finding the exact mechanism responsible for developing myelopathy in such individuals.

MRI of the spine could not be performed before or after the treatment to demonstrate myelopathy because of the cost constraint. This was the only limitation for this case report.

\section{Acknowledgements \\ Conflicts of interest \\ There are no conflicts of interest.}

\section{References}

1 Usha Menon V, Sundaram KR, Unnikrishnan AG, Jayakumar RV, Nair V, Kumar $\mathrm{H}$. High prevalence of undetected thyroid disorders in an iodine sufficient adult south Indian population. J Indian Med Assoc 2009; 107:72-77.

2 Wise MP, Blunt S, Lane RJM. Neurological presentations of hypothyroidism: the importance of slow relaxing reflexes. J R Soc Med 1995; 88:272-274.

3 Brain L, Jellinek EH, Ball K. Hashimoto's disease and encephalopathy. Lancet 1966; ii:512-514.

4 Azuma T, Uemichi T, Funauchi M, Doi S, Matsubara T. Myelopathy associated with Hashimoto's disease. J Neurol Neurosurg Psychiatry 2000; 68:679-683. 